\title{
Einstein-Maxwell-scalar black holes: classes of solutions, dyons and extremality
}

\section{Astefanesei, ${ }^{a}$ C. Herdeiro, ${ }^{b, c}$ A. Pombo ${ }^{d}$ and E. Radu ${ }^{d}$}

${ }^{a}$ Pontificia Universidad Católica de Valparaíso, Instituto de Física, Av. Brasil 2950, Valparaíso, Chile

${ }^{b}$ CENTRA, Departamento de Física, Instituto Superior Técnico - IST, Universidade de Lisboa - UL, Avenida Rovisco Pais 1, 1049 Lisboa, Portugal

${ }^{c}$ Departamento de Matemática da Universidade de Aveiro and CIDMA, Campus de Santiago, 3810-183 Aveiro, Portugal

${ }^{d}$ Departamento de Fúsica da Universidade de Aveiro and CIDMA, Campus de Santiago, 3810-183 Aveiro, Portugal

E-mail: dumitru.astefanesei@pucv.cl, herdeiro@ua.pt, pomboalexandremira@ua.pt, eugen.radu@ua.pt

ABSTRACT: Spherical black hole (BH) solutions in Einstein-Maxwell-scalar (EMS) models wherein the scalar field is non-minimally coupled to the Maxwell invariant by some coupling function are discussed. We suggest a classification for these models into two classes, based on the properties of the coupling function, which, in particular, allow, or not, the ReissnerNordström (RN) BH solution of electrovacuum to solve a given model. Then, a comparative analysis of two illustrative families of solutions, one belonging to each class is performed: dilatonic versus scalarised BHs. By including magnetic charge, that is considering dyons, we show that scalarised BHs can have a smooth extremal limit, unlike purely electric or magnetic solutions. In particular, we study this extremal limit using the entropy function formalism, which provides insight on why both charges are necessary for extremal solutions to exist.

Keywords: Black Holes, Classical Theories of Gravity

ARXIV EPRINT: 1905.08304 


\section{Contents}

1 Introduction 1

2 The EMS model 3

2.1 The action and equations of motion 3

2.2 The coupling function and a classification of EMS models 4

2.3 Spontaneous scalarisation of dyonic RN BHs: zero modes 7

3 Non-extremal black holes $\quad 8$

$\begin{array}{ll}3.1 & \text { The ansatz and field equations } \\ 3.2 & 8\end{array}$

$\begin{array}{lr}3.2 & \text { Asymptotic forms of the solutions }\end{array}$

$\begin{array}{ll}3.3 \text { Quantities of interest and Smarr law } & 10\end{array}$

$\begin{array}{lll}3.4 & \text { The BH solutions } & 11\end{array}$

$\begin{array}{lll}3.4 .1 & \text { The purely electric BHs } & 11\end{array}$

$\begin{array}{lll}3.4 .2 & \text { The dyonic BHs } & 13\end{array}$

4 Extremal BHs $\quad 14$

4.1 Numerical construction 14

4.2 An analytic approach: the attractor mechanism and entropy function $\quad 16$

$\begin{array}{llr}5 & \text { Discussion } & 18\end{array}$

$\begin{array}{ll}\text { A Exact solutions with a linear coupling } & 21\end{array}$

A.1 Purely electric BHs 21

A.2 Dyonic BHs 22

$\begin{array}{lll}\text { A.2.1 } \alpha=1 & 22\end{array}$

$\begin{array}{lll}\text { A.2.2 } \alpha=\sqrt{3} & 22\end{array}$

\section{Introduction}

Einstein-Maxwell (EM) theory, a.k.a. electrovacuum, is the quintessential source-free, gravitational relativistic field theory. Its static physical black holes (BHs) belong to the 3-parameter Reissner-Nordström (RN) family, described by mass $M$, electric $Q$ and magnetic $P$ charges. These BHs are perturbatively stable [1,2], and, for given $M$, can only sustain charges $(P, Q)$ if $\sqrt{Q^{2}+P^{2}} \leqslant M$. When the equality holds, the extremal limit is attained. Extremal RN BHs are special. They are non-singular spacetimes, on and outside a degenerate and $C^{\infty}$ smooth event horizon, that: (i) have a vanishing Hawking temperature and are BPS states that possess Killing spinors when embedded in supergravity [3]; (ii) have a near horizon geometry which is, itself, a solution of the EM theory [4] - the Robinson-Bertotti $\left(A d S_{2} \times S^{2}\right)$ vacuum [5, 6]; and (iii) allow a no-force condition and a multi-BH generalisation, described by the Majumdar-Papapetrou metrics [7-9]. 
A simple and natural generalisation of the EM theory is to consider an additional dynamical real scalar field, with a standard kinetic term. A variety of such EM-scalar (EMS) models are possible, depending on the way the scalar field couples to the Maxwell field. ${ }^{1}$ Remarkably, if the scalar field is minimally coupled to the Maxwell, no new charged $\mathrm{BH}$ solutions are possible, beyond $\mathrm{RN}$, even if the scalar field is allowed to have a nonnegative self-interactions potential [10]. In these conditions, charged BHs cannot have scalar hair. Quite different possibilities, however, arise if a non-minimal coupling between the scalar and Maxwell field is allowed. This is the case we shall be interested in this paper.

The first such non-minimally coupled EMS model emerged in the pioneering unification theory of Kaluza [11] and Klein [12], soon after Einstein constructed General Relativity (GR) [13]. These EMS models turned out to be ubiquitous in the four dimensional description of higher dimensional GR-inspired theories [14], as well as in supergravity, see e.g. [15]. In the former, as well as in the latter with a higher dimensional origin, the scalar field describes how the extra dimension(s) dilate along the four dimensional spacetime, being dubbed dilaton. The dilaton has a specific non-minimal coupling with the Maxwell term in the EMS action. This coupling prevents EM theory to be a consistent truncation of this class of EM-dilaton models. In particular, the RN solution of EM theory does not solve these EM-dilaton models. Instead, new charged BHs with a non-trivial scalar field profile exist [16, 17], which are known in closed analytic form and that present RN-unlike features. For instance, the BH charge to mass ratio can exceed unity (see, e.g. [18]). As another example, there are no extremal BHs with a regular horizon in the purely electric (or purely magnetic) case. These limiting solutions become naked singularities, ${ }^{2}$ a sharp contrast with the physically interesting extremal RN BH. Nonetheless, dilatonic BHs provided an example of asymptotically flat charged BHs with scalar hair [22], albeit of secondary type [23].

Once embedded in string theory, the dilaton $\phi$ controls the string coupling, which is related to the vacuum expectation of the asymptotic value of the dilaton, $g_{s}=e^{\left\langle\phi_{\infty}\right\rangle}$. Therefore, a consistent analysis of hairy BHs in string theory should consider a dynamical dilaton whose asymptotic value can vary [24] (see, also, [25] for a resolution of the appearance of the scalar charges in the first law of thermodynamics). This need, however, is mitigated by the attractor mechanism [26, 27]: the near horizon data (particularly, the entropy) of extremal $\mathrm{BHs}$ is independent of the asymptotic values of the moduli. The mechanism is based on a simple physical intuition; when the temperature vanishes, there is a symmetry enhanced near horizon geometry: $A d S_{2} \times S^{2}$. The infinite long throat of $A d S_{2}$ yields the decoupling between the physics at the boundary from the physics at the extremal horizon [28]. A similar decoupling plays a central role in the $A d S / C F T$ duality (see, e.g. [29, 30]).

EMS models with more generic non-minimal couplings (than the dilatonic one) between the scalar field and the Maxwell term are also of interest. For example, such models

\footnotetext{
${ }^{1}$ Herein we shall always consider that the scalar field is minimally coupled to gravity.

${ }^{2}$ Asymptotically flat, purely electric BHs exist also in EMS models with a non-trivial scalar potential, explicit solutions being reported in $[19,20]$. Since in this case there are two different terms that source the scalar field (a self-interaction potential and the term coming from the non-trivial coupling with the electric field), a balance is possible, which allows for a well defined extremal limit [21].
} 
were considered in cosmological inflationary scenarios [31, 32]. In the context of BHs, it was recently realised that a family of couplings can trigger a spontaneous scalarisation of the RN BH [33, 34]. In this class of EMS models, unlike the aforementioned dilatonic models, the RN BH is a solution. For sufficiently large charge to mass ratio, however, the RN BH becomes unstable against scalar perturbations and dynamically grows a scalar field profile; it becomes energetically favourable to scalarise. The hair growth stalls due to non-linear effects leading to a scalarised $\mathrm{BH}$ (to be distinguished from dilatonic $\mathrm{BH}$ ). The fundamental scalarised charged BHs, which are the ones formed dynamically are, moreover, perturbatively stable [38-40] and therefore represent the endpoint of the non-linear evolution of the unstable RN BHs. Consequently, these scalarised BHs are an example of dynamically grown scalar hair.

The scalarised BHs studied up to now contain only electric charge. They possess no extremal limit. Rather, a critical solution is attained for the maximal charge a $\mathrm{BH}$ can support, which (numerical evidence suggests) is singular. This parallels the status of dilatonic BHs. For the latter, however, the introduction of an additional magnetic charge leads to dyonic BHs with an extremal (non-singular) limit, which have been constructed for specific couplings [41-43]. Given the importance of extremal solutions, it is interesting to inquire which are the properties of the family of dyonic scalarised BHs and, in particular, of their extremal limit.

In fact, the considerations above suggest a comparison between dilatonic and scalarised BHs can be instructive. The purpose of this paper is to perform such a comparison, for the canonical dilatonic coupling and the reference model of scalarised solutions introduced in [33]. Our results, within this comparative study, include: $(i)$ the introduction of a general framework to study EMS for any scalar non-minimal coupling; ( $i i)$ the first study of dyonic scalarised BHs; (iii) establishing that extremal scalarised BHs indeed exist (only) when both electric and magnetic charges are present; and $(i v)$ the study of the corresponding near horizon geometries via the attractor and entropy function formalism of [44-46].

This paper is organised as follows. In section 2 we present the EMS models and propose a classification of the $\mathrm{BH}$ solutions, based on the behaviour of the coupling function. We also derive the zero mode of the RN BHs for the models that allow BH scalarisation. Section 3 contains a discussion of the non-extremal BHs for both two classes of solutions (dilatonic and scalarised). In section 4, we study the extremal BHs toghether with the corresponding near horizon geometries, using the attractors formalism. We conclude in section 5 with a discussion and some further remarks. The appendix contains a brief review of the known exact solutions, all of which occur for a dilatonic coupling.

\section{The EMS model}

\subsection{The action and equations of motion}

The EMS family of models is defined by the following action (we set $c=G=4 \pi \epsilon_{0}=1$ )

$$
\mathcal{S}=\frac{1}{16 \pi} \int d^{4} x \sqrt{-g}\left(R-2 \partial_{\mu} \phi \partial^{\mu} \phi-f(\phi) F_{\mu \nu} F^{\mu \nu}\right),
$$

where $R$ is the Ricci scalar, $F_{\mu \nu}=\partial_{\mu} A_{\nu}-\partial_{\nu} A_{\mu}$ is the Maxwell field and $\phi$ is the scalar field. The coupling function $f(\phi)$ governs the non-minimal coupling of $\phi$ to the electromag- 
netic field. From the outset we are excluding an axion-type coupling of the scalar to the electromagnetic field, as well as any sort of self-interaction of the scalar field. The model may, of course, be generalised in these directions.

The field equations obtained by varying the above action principle with respect to the field variables $g_{\mu \nu}, \phi$ and $A_{\mu}$ are

$$
\begin{aligned}
R_{\mu \nu}-\frac{1}{2} R g_{\mu \nu} & =2\left[\partial_{\mu} \phi \partial_{\nu} \phi-\frac{1}{2} g_{\mu \nu} \partial_{\rho} \phi \partial^{\rho} \phi+f(\phi)\left(F_{\mu \rho} F_{\nu}{ }^{\rho}-\frac{1}{4} g_{\mu \nu} F_{\rho \sigma} F^{\rho \sigma}\right)\right], \\
\frac{1}{\sqrt{-g}} \partial_{\mu}\left(\sqrt{-g} \partial^{\mu} \phi\right) & =\frac{1}{4} \frac{d f(\phi)}{d \phi} F_{\rho \sigma} F^{\rho \sigma} \\
\partial_{\mu}\left(\sqrt{-g} f(\phi) F^{\mu \nu}\right) & =0
\end{aligned}
$$

An ansatz suitable to address both the (generic) asymptotically flat solutions and the Robinson-Bertotti (near horizon) geometries reads

$$
d s^{2}=-a(r)^{2} d t^{2}+b(r)^{2}\left(d \theta^{2}+\sin ^{2} \theta d \varphi^{2}\right)+c(r)^{2} d r^{2} .
$$

The gauge 4-potential ansatz compatible with the symmetries of (2.5) contains an electric potential $V(r)$ and a magnetic term,

$$
A=V(r) d t+P \cos \theta d \varphi
$$

where $P=$ constant is the magnetic charge. The scalar field is a function of $r$ only, $\phi \equiv \phi(r)$.

The Maxwell equation (2.4) yields a first integral

$$
V^{\prime}=\frac{a c}{b^{2}} \frac{Q}{f(\phi)},
$$

where $Q=$ constant is the electric charge (measured at infinity), and henceforth a prime denotes a derivative w.r.t. the radial coordinate $r$.

The equations of motion (2.2)-(2.4) are invariant under the electro-magnetic duality transformation

$$
\{P \rightarrow Q, \quad Q \rightarrow P\} \text { and } f(\phi) \rightarrow 1 / f(\phi)
$$

In what follows, we shall assume, without any loss of generality, that both $Q$ and $P$ are positive and that

$$
Q \geqslant P
$$

such that for scalarised BHs, the (electric) solutions in [33, 34] are recovered as $P \rightarrow 0$.

\subsection{The coupling function and a classification of EMS models}

The RN BH is a solution of $(2.2)-(2.4)$ with $f(\phi)=1, \phi=$ constant and

$$
V(r)=-\frac{Q}{r}, \quad a(r)^{2}=\frac{1}{c(r)^{2}}=1-\frac{2 M}{r}+\frac{Q^{2}+P^{2}}{r^{2}}, \quad b(r)=r,
$$


where $M$ is the $\mathrm{BH}$ ADM mass. For a more general $f(\phi)$ the RN BH may or may not solve (2.2)-(2.4). This naturally leads to two classes of EMS models. (Note that, in this classification, we assume, without any loss of generality, that the scalar field vanishes asymptotically, $\phi(r) \stackrel{r \rightarrow \infty}{\longrightarrow} 0$.)

Class I or dilatonic-type. In this class of EMS models $\phi(r)=0$ does not solve the field equations. ${ }^{3}$ Thus RN is not a solution. Then, the scalar field equation (2.3) implies that

$$
\left.f_{, \phi}(0) \equiv \frac{d f(\phi)}{d \phi}\right|_{\phi=0} \neq 0
$$

A representative example of coupling for this class is the standard dilatonic coupling

$$
f(\phi)=e^{2 \alpha \phi},
$$

in which case we refer to $\phi$ is a dilaton field. The arbitrary nonzero constant $\alpha$ is taken to be positive without any loss of generality. Indeed, the solutions remain invariant under the simultaneous sign change $(\alpha, \phi) \rightarrow-(\alpha, \phi)$. Thus, flipping the sign of $\alpha$ simply corresponds to flipping the sign of $\phi$. The coupling (2.12) appears naturally in Kaluza-Klein models and supergravity/low-energy string theory models. Three reference values for the coupling constant $\alpha$ in (2.12) are:

$$
\alpha=0 \text { (EM theory) } \quad \alpha=1 \text { (low energy strings) } \quad \alpha=\sqrt{3} \text { (KK theory). }
$$

Some exact, closed form BH solutions of (2.2)-(2.4) with (2.12) are known and presented in appendix A. Other exact solution examples in this class (with a nondilatonic coupling) are given in [47].

Class II or scalarised-type. In this class of EMS models $\phi(r)=0$ solves the field equations. Thus RN is a solution. This demands that

$$
\left.f_{, \phi}(0) \equiv \frac{d f(\phi)}{d \phi}\right|_{\phi=0}=0 .
$$

This condition is naturally implemented, for instance, if one requires the model to be $\mathbb{Z}_{2}$-invariant under $\phi \rightarrow-\phi$. The $\mathrm{RN}$ solution, however, is (in general) not unique. These EMS models may contain a second set of BH solutions, with a nontrivial scalar field profile - the scalarised BHs. Below some conditions for this to occur are discussed. Such second set of BH solutions may, or may not, continuously connect with RN BHs. This leads to two subclasses.

- Subclass IIA or scalarised-connected-type. In this subclass of EMS models, the scalarised BHs bifurcate from RN BHs, and reduce to the latter for $\phi=0$. This bifurcation moreover, may be associated to a tachyonic instability, against scalar

\footnotetext{
${ }^{3}$ There is an exceptional case: if $Q=P, \phi=0$ solves this class, so that the dyonic, equal charges RN $\mathrm{BH}$ is a solution.
} 
perturbations, of the RN BH. Considering a small- $\phi$ expansion of the coupling function

$$
f(\phi)=f(0)+\left.\frac{1}{2} \frac{d^{2} f(\phi)}{d \phi^{2}}\right|_{\phi=0}+\ldots,
$$

equation (2.3) linearised for small- $\phi$ reads:

$$
\left(\square-\mu_{\text {eff }}^{2}\right) \phi=0, \quad \text { where } \mu_{\text {eff }}^{2}=\left.\frac{F_{\mu \nu} F^{\mu \nu}}{4} \frac{d^{2} f(\phi)}{d \phi^{2}}\right|_{\phi=0} .
$$

The instability arises if $\mu_{\text {eff }}^{2}<0$, which in particular requires

$$
\left.f_{, \phi \phi}(0) \equiv \frac{d^{2} f(\phi)}{d \phi^{2}}\right|_{\phi=0} \neq 0,
$$

and with the opposite sign of $F_{\mu \nu} F^{\mu \nu}$. A reference example of a coupling function in this subclass, which we consider in this work is [33]

$$
f(\phi)=e^{2 \alpha \phi^{2}}
$$

a case which is also relevant in cosmology [31, 32]. Depending on the coupling, this subclass could also contain another family of disconnected scalarised BHs, akin to the ones of class IIB below.

- Subclass IIB or scalarised-disconnected-type. In this subclass of EMS models, the scalarised BHs do not bifurcate from RN BHs, and do not reduce to the latter for $\phi=0$. This is the case if there is no tachyonic instability of RN, for which a sufficient (but not necessary) condition is that

$$
\left.f_{, \phi \phi}(0) \equiv \frac{d^{2} f(\phi)}{d \phi^{2}}\right|_{\phi=0}=0 \text {. }
$$

We shall not address further this case in this paper (which, moreover, was not considered yet in the literature), but a representative coupling would be, say, $f(\phi)=1+\alpha \phi^{4}$.

Condition (2.14) guarantees $\mathrm{RN}$ is a solution. But it does not guarantee the existence of scalarised BHs. In the case of purely electric (or magnetic) BHs, two Bekenstein-type identities can be derived, which put some constraints on $f(\phi)$ so that scalarised solutions exist. These can be derived as follows.

To derive the first identity, the scalar field equation (2.3) is multiplied by $f_{, \phi}$ and integrated over a spacetime volume. Integrating by parts and discarding the boundary terms, by virtue of the horizon properties and asymptotic flatness, one obtains

$$
\int d^{4} x \sqrt{-g}\left(f_{, \phi \phi} \partial_{\mu} \phi \partial^{\mu} \phi+\frac{f_{, \phi}^{2}}{4} F^{2}\right)=0 \text {. }
$$

The sign of neither term is fixed, in general, and specific considerations are required. For instance, a purely electric field has $F^{2}<0$; this implies

$$
f_{, \phi \phi}>0,
$$


must hold for some range of the radial coordinate $r$, otherwise the two terms of the integrand in (2.20) will have always the same sign, making the identity only possible for $\phi=0$.

A second identity is found by multiplying (2.3) by $\phi$, which results, via a similar procedure, in

$$
\int d^{4} x \sqrt{-g}\left(\partial_{\mu} \phi \partial^{\mu} \phi+\frac{\phi f_{, \phi}}{4} F^{2}\right)=0
$$

This imples that for a purely electric field the potential should satisfy the condition

$$
\phi f_{, \phi}>0,
$$

for some range of $r$. Similar arguments hold for purely magnetic solutions, which implies $f_{, \phi \phi}<0$ and $\phi f_{, \phi}<0$, respectively. No such results can be established in the generic dyonic case, since the sign of $F^{2}=F_{\mu \nu} F^{\mu \nu}$ is not determined, a priori.

\subsection{Spontaneous scalarisation of dyonic RN BHs: zero modes}

Class IIA of EMS models is particularly interesting because it accommodates the dynamical phenomenon of spontaneous scalarisation [33, 34] (see also [35-37] for earlier discussions of charged BHs scalarisation in different models). At the linear level this is manifest in the tachyonic instability (2.16). For a dyonic RN BHs (2.10), $F_{\mu \nu} F^{\mu \nu}=-2\left(Q^{2}-P^{2}\right) / r^{4}$. Thus, under the assumption (2.9) a tachyonic instability requires $f_{, \phi \phi}(0)>0$. Let us study this instability, generalising the analysis in $[33,34]$ for the dyonic RN case.

Assuming separation of variables,

$$
\phi=Y_{\ell m}(\theta, \varphi) U(r),
$$

where $Y_{\ell m}$ are the real spherical harmonics and $\ell, m$ are the associated quantum numbers, i.e. $\ell=0,1, \ldots$ and $-\ell \leqslant m \leqslant \ell$, the equation for the radial amplitude $U(r)$ reads

$$
\left(\frac{r^{2} U^{\prime}}{c(r)^{2}}\right)^{\prime}=\left[\ell(\ell+1)+\frac{\left(P^{2}-Q^{2}\right)}{2 r^{2}} f_{, \phi \phi}(0)\right] U,
$$

where $c(r)$ is given by (2.10). Observe that the term $\mu_{\text {eff }}^{2}=\left(P^{2}-Q^{2}\right) f_{, \phi \phi}(0) / r^{2}$ acts as the effective mass for the perturbations and the condition $\mu_{\text {eff }}^{2}<0$ requires $f_{, \phi \phi}(0)>0$, as discussed above.

For spherically symmetric perturbations $\ell=0$, and eq. (2.25) possesses an exact solution which is regular on and outside the horizon and vanishes at infinity ${ }^{4}$

$$
U(r)=P_{u}\left(1+\frac{2\left(Q^{2}-P^{2}\right)\left(r-r_{H}\right)}{r\left(r_{H}^{2}+P^{2}-Q^{2}\right)}\right), \text { where } u=\frac{1}{2}\left(\sqrt{1-2 f_{, \phi \phi}(0)}-1\right),
$$

where $r_{H}$ is the event horizon radial coordinate and $P_{u}$ is a Legendre function. This solution is physical for $f_{, \phi \phi}(0)>1 / 2$, a condition which, for the coupling function (2.18) implies

$$
\alpha>1 / 8
$$

\footnotetext{
${ }^{4}$ The limit $P=0$ of this solution has been discussed in [34].
} 

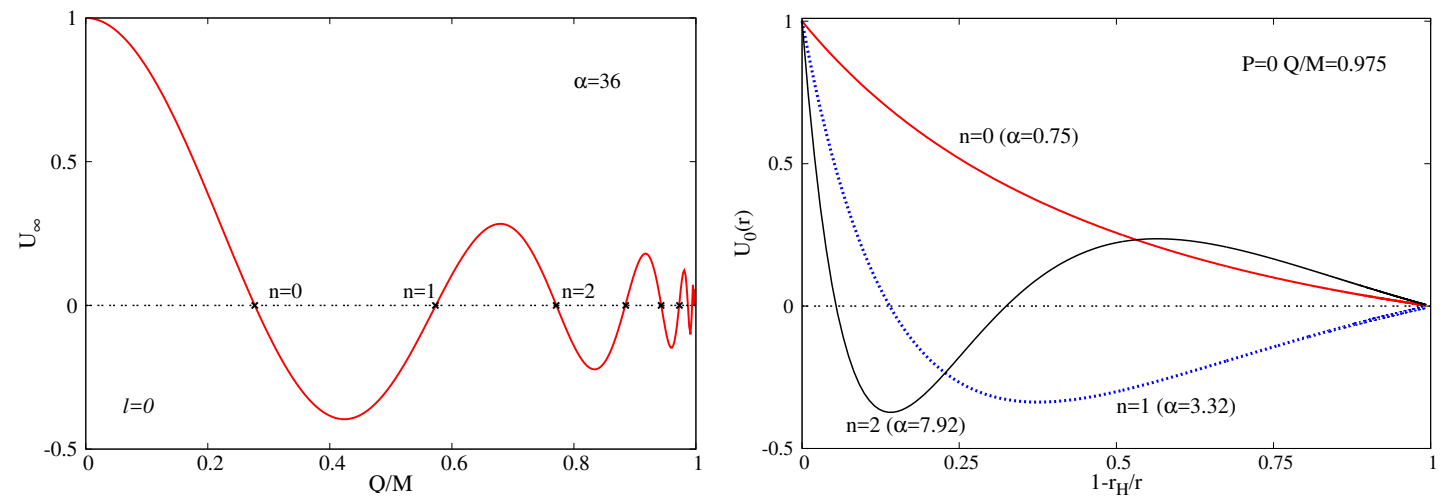

Figure 1. (Left panel) The asymptotic value $U_{\infty}$ of the zero-mode amplitude $U$ for $\alpha=36$ as a function of the charge to mass ratio of a RN BH. An infinite set of configurations with $U_{\infty}=0$ exist, labelled by $n$, the number of nodes of $U(r)$. (Right panel) The profiles of three zero mode amplitudes $U(r)$ with a different node number, for a given $\mathrm{RN}$ background.

For generic parameters $\left(f_{, \phi \phi}(0), Q, P, r_{H}\right)$, the function $U(r)$ approaches a constant non zero value as $r \rightarrow \infty$,

$$
U(r) \rightarrow U_{\infty}={ }_{2} F_{1}\left[\frac{1-\sqrt{1-2 f_{, \phi \phi}(0)}}{2}, \frac{1+\sqrt{1-2 f_{, \phi \phi}(0)}}{2}, 1 ; \frac{Q^{2}-P^{2}}{Q^{2}-P^{2}-r_{H}^{2}}\right]+\mathcal{O}\left(\frac{1}{r}\right) .
$$

Thus finding the $\ell=0$ unstable mode of a $\mathrm{RN} \mathrm{BH}$ with given $P, Q, M$ reduces to a study of the zeros of the hypergeometric function ${ }_{2} F_{1}$, so that $U_{\infty}=0$.

The value of $U_{\infty}$ for the coupling function (2.18) and an illustrative value of $\alpha$ is shown in figure 1 (left panel). Therein, the integer $n$ labels the number of nodes of the function $U(r)$ when the correct boundary condition at infinity is met: given a RN background, the solutions with $U_{\infty}=0$ are found for a discrete sequence $\alpha_{n}$, each one corresponding to a different node number, cf. figure 1 (right panel). To simplify the picture, the results in figure 1 correspond to $P=0$; a similar pattern holds also in the dyonic case.

The solution (2.26) yields a dyonic RN BH surrounded by a vanishingly small scalar field. The set of such RN BHs (varying $\alpha$ ) constitutes the existence line, the branching line between the RN and scalarised BHs. The latter are the non-linear continuation of the (infinitesimally small) scalar clouds, i.e. the solutions of eq. (2.25). As remarked above, these clouds are labelled by three integer numbers $(\ell, m, n)$. In what follows, however, we shall restrict our study to the simplest case of nodeless, spherically symmetric configurations. More general configurations in the purely electric case have been discussed in [33, 34].

\section{Non-extremal black holes}

Let us now construct, numerically, the non-linear BH solutions, for both class I and IIA, starting with the non-extremal BHs.

\subsection{The ansatz and field equations}

In the numerical study of the solutions, it is convenient to work in Schwarzschild-like coordinates, with a metric gauge choice $b(r)=r, a(r)^{2}=e^{-2 \delta(r)} N(r)$ and $c(r)^{2}=1 / N(r)$. 
Then, (2.5) becomes:

$$
d s^{2}=-e^{-2 \delta(r)} N(r) d t^{2}+\frac{d r^{2}}{N(r)}+r^{2}\left(d \theta^{2}+\sin ^{2} \theta d \varphi^{2}\right), \quad \text { where } N(r) \equiv 1-\frac{2 m(r)}{r} .
$$

The function $m(r)$ corresponding to a local mass function, known as the Misner-Sharp mass [48].

The equations of motion (2.2) and (2.3), together with the first integral (2.7) implies that the functions $m, \sigma, \phi$ solve the ordinary differential equations

$$
\begin{aligned}
& m^{\prime}=\frac{1}{2} r^{2} N \phi^{\prime 2}+\frac{1}{2 r^{2}}\left(\frac{Q^{2}}{f(\phi)}+f(\phi) P^{2}\right), \\
& \delta^{\prime}+r \phi^{\prime 2}=0, \\
& \left(e^{-\delta} r^{2} N \phi^{\prime}\right)^{\prime}+\frac{e^{-\delta}}{2 r^{2} f(\phi)} \frac{d f(\phi)}{d \phi}\left(\frac{Q^{2}}{f(\phi)}-f(\phi) P^{2}\right)=0,
\end{aligned}
$$

which can also be derived from the following effective action:

$$
S_{\mathrm{eff}}=\int d t d r\left[e^{-\delta} m^{\prime}-\frac{1}{2} e^{-\delta} r^{2} N \phi^{2}+\frac{f(\phi)}{2}\left(r^{2} e^{\delta} V^{\prime 2}-\frac{e^{-\delta}}{r^{2}} P^{2}\right)\right],
$$

while $V^{\prime}=e^{-\delta} Q /\left(r^{2} f(\phi)\right)$. The Einstein equations also yield a constraint equation,

$$
\frac{1}{2} N^{\prime \prime}-N \delta^{\prime \prime}+N^{\prime}\left(\frac{1}{r}-\frac{3}{2} \delta^{\prime}\right)+N \delta^{\prime}\left(\delta^{\prime}-\frac{1}{r}\right)+N \phi^{\prime 2}-\frac{1}{r^{4}}\left[\frac{Q^{2}}{f(\phi)}+P^{2} f(\phi)\right]=0,
$$

which can be shown to be a linear combination of equations (3.2)-(3.4) together with the first derivatives of (3.2)-(3.3). It is also of interest to observe that equations (3.2)-(3.4) possess the first integral

$$
e^{-2 \delta} r^{4} N\left[1+\frac{N \delta^{\prime}}{r}-N\left(\frac{N^{\prime}}{2 N}-\delta^{\prime}\right)^{2}+\frac{1}{r^{4}}\left(\frac{Q^{2}}{f(\phi)}+P^{2} f(\phi)\right)\right]=u_{0},
$$

where the constant $u_{0}$ is fixed by the asymptotics.

To assess possible singular behaviours we remark that the expression of the Ricci and Kretschmann scalars for the line-element (3.1) read:

$$
\begin{aligned}
& R=\frac{N^{\prime}}{r}\left(3 r \delta^{\prime}-4\right)+\frac{2}{r^{2}}\left\{1+N\left[r^{2} \delta^{\prime \prime}-\left(1-r \delta^{\prime}\right)^{2}\right]\right\}-N^{\prime \prime}, \\
& K=\frac{4}{r^{4}}(1-N)^{2}+\frac{2}{r^{2}}\left[N^{\prime 2}+\left(N^{\prime}-2 N \delta^{\prime}\right)^{2}\right]+\left[N^{\prime \prime}-3 \delta^{\prime} N^{\prime}+2 N\left(\delta^{\prime 2}-\delta^{\prime \prime}\right)\right]^{2} .
\end{aligned}
$$

\subsection{Asymptotic forms of the solutions}

To construct $\mathrm{BH}$ solutions, we assume the existence of a horizon located at $r=r_{H}>0$. In its exterior neighbourhood, one finds the following approximate solution, valid for nonextremal BHs:

$$
\begin{aligned}
m(r) & =\frac{r_{H}}{2}+m_{1}\left(r-r_{H}\right)+\ldots, & \delta(r) & =\delta_{0}+\delta_{1}\left(r-r_{H}\right)+\ldots, \\
\phi(r) & =\phi_{0}+\phi_{1}\left(r-r_{H}\right)+\ldots, & V(r) & =v_{1}\left(r-r_{H}\right)+\ldots,
\end{aligned}
$$


where out of the six parameters, $m_{1}, \delta_{0}, \delta_{1}, \phi_{0}, \phi_{1}, v_{1}$, only two are essential, $\phi_{0}$ and $\delta_{0}$, the remaining being determined in terms of these and the global charges as:

$$
\begin{aligned}
m_{1} & =\frac{1}{2 r_{H}^{2}}\left[\frac{Q^{2}}{f\left(\phi_{0}\right)}+f\left(\phi_{0}\right) P^{2}\right], & \phi_{1} & =\left.\frac{d f(\phi)}{d \phi}\right|_{\phi_{0}} \frac{1}{2 r_{H}} \frac{\left[\frac{Q^{2}}{f\left(\phi_{0}\right)}-f\left(\phi_{0}\right) P^{2}\right]}{\left[\frac{Q^{2}}{f\left(\phi_{0}\right)}+f\left(\phi_{0}\right) P^{2}-r_{H}^{2}\right]}, \\
\delta_{1} & =-r_{H} \phi_{1}^{2}, & v_{1} & =-\frac{e^{-\delta_{0}} Q}{r_{H}^{2} f\left(\phi_{0}\right)} .
\end{aligned}
$$

Note that a similar result holds when considering higher order terms in the approximate solution (3.9).

For large $r$, one finds the following asymptotic expansions:

$$
\begin{array}{ll}
m(r)=M-\frac{Q^{2}+P^{2}+Q_{s}^{2}}{2 r}+\ldots, & \phi(r)=\frac{Q_{s}}{r}+\ldots, \\
V(r)=\Phi_{e}-\frac{Q}{r}+\ldots, & \delta(r)=\frac{Q_{s}^{2}}{2 r^{2}}+\ldots
\end{array}
$$

The essential parameters introduced in the expansion at infinity (3.11) are the ADM mass $M$, electric and magnetic charges $Q, P$, electrostatic potential at infinity $\Phi_{e}$ and scalar 'charge' $Q_{s}$.

The Ricci scalar (3.8) vanishes as $r \rightarrow r_{H}$, while the Kretschmann scalar (3.9) reads

$$
K=\frac{12}{r_{H}^{4}}\left\{1-\frac{2}{r_{H}^{2}}\left[\frac{Q^{2}}{f(\phi)}+f(\phi) P^{2}\right]+\frac{5}{3 r_{H}^{4}}\left[\frac{Q^{2}}{f(\phi)}+f(\phi) P^{2}\right]^{2}\right\}+\mathcal{O}\left(r-r_{H}\right) .
$$

\subsection{Quantities of interest and Smarr law}

Two horizon physical quantities of interest are the Hawking temperature and horizon area

$$
T_{H}=\frac{1}{4 \pi} N^{\prime}\left(r_{H}\right) e^{-\delta_{0}}, \quad A_{H}=4 \pi r_{H}^{2} ;
$$

these, together with the horizon scalar field value $\phi_{0}$ compose the relevant horizon data.

The Smarr-like relation [49] for this family of models turns out to have no explicit imprint of the scalar hair,

$$
M=\frac{1}{2} T_{H} A_{H}+\Phi_{e} Q+\Phi_{m} P
$$

where we have defined a 'magnetic' potential as $\Phi_{m} \equiv \int_{r_{H}}^{\infty} d r e^{-\delta} f(\phi) P / r^{2}$. One can then compute a first law of $\mathrm{BH}$ thermodynamics for EMS BHs, that reads:

$$
d M=\frac{1}{4} T_{H} d A_{H}+\Phi_{e} d Q+\Phi_{m} d P .
$$

A non-linear Smarr relation (i.e. mass formula) can also be established for this family of models,

$$
M^{2}+Q_{s}^{2}=Q^{2}+P^{2}+\frac{1}{4} A_{H}^{2} T_{H}^{2}
$$


which is derived by evaluating the expression of the first integral (3.7) at the horizon and at infinity, for the approximate form of the solutions (3.9) and (3.11), respectively.

Finally, one can prove that the solutions satisfy the virial identity, which is obtained by a Derrick-type [50] scaling argument, see e.g. [23]

$$
\int_{r_{H}}^{\infty} d r\left\{e^{-\delta} \phi^{\prime 2}\left[1+\frac{2 r_{H}}{r}\left(\frac{m}{r}-1\right)\right]\right\}=\int_{r_{H}}^{\infty} d r\left\{e^{-\delta}\left(1-\frac{2 r_{H}}{r}\right) \frac{1}{r^{2}}\left[\frac{Q^{2}}{f(\phi)}+f(\phi) P^{2}\right]\right\} .
$$

One can show that $1+\frac{2 r_{H}}{r}\left(\frac{m}{r}-1\right)>0$, i.e. the left hand side integrand, is strictly positive. Thus, the virial identity shows that a nontrivial scalar field requires a nonzero electric/magnetic charge so that the right hand side is nonzero.

The model possesses the scaling symmetry

$$
r \rightarrow \lambda r \quad(P, Q) \rightarrow \lambda(P, Q)
$$

where $\lambda>0$ is a constant. Under this scaling symmetry, all other quantities change accordingly, e.g., $M \rightarrow \lambda M$, while the coupling function $f(\phi)$ is unchanged. Thus, for a physical discussion, we consider quantities which are invariant under the transformation (3.18). Consequently, we introduce the standard reduced quantities

$$
q \equiv \frac{\sqrt{Q^{2}+P^{2}}}{M}, \quad a_{H} \equiv \frac{A_{H}}{16 \pi M^{2}}, \quad t_{H} \equiv 8 \pi T_{H} M
$$

For example, dyonic RN BHs have closed expressions for $a_{H}, t_{H}$ :

$$
a_{H}^{(\mathrm{RN})}=\frac{1}{4}\left(1+\sqrt{1-q^{2}}\right)^{2}, \quad t_{H}^{(\mathrm{RN})}=\frac{4 \sqrt{1-q^{2}}}{\left(1+\sqrt{1-q^{2}}\right)^{2}} .
$$

In appendix A we exhibit the corresponding expressions for other dilatonic BHs known in closed analytic form, which are class I solutions.

The generic dilatonic dyonic solutions are not known in closed form, which hold also for all scalarised BHs. These solutions are found numerically, by matching the asymptotics (3.9), (3.11). Equations (3.2)-(3.4) are solved by using a standard Runge-Kutta ODE solver and implementing a shooting method in terms of the parameters $\phi_{0}, \delta_{0}$.

\subsection{The BH solutions}

\subsubsection{The purely electric BHs}

Let us start with our reference class I solutions. The behaviour of the dilatonic BHs with any $\alpha>0$ is rather similar, albeit $\alpha=1$ is a somewhat special point that separates the family into two subsets with respect to the behaviour of some physical quantities. This can be seen from the study of the exact solutions in appendix A. For any given $\alpha$, the branch of dilatonic BHs bifurcates from the Schwarzschild BH $(q=0)$, rather than RN BHs, and ends in a critical solution which is approached for a certain maximal $q$

$$
q_{\max }^{(\mathrm{D})}=\sqrt{1+\alpha^{2}},
$$



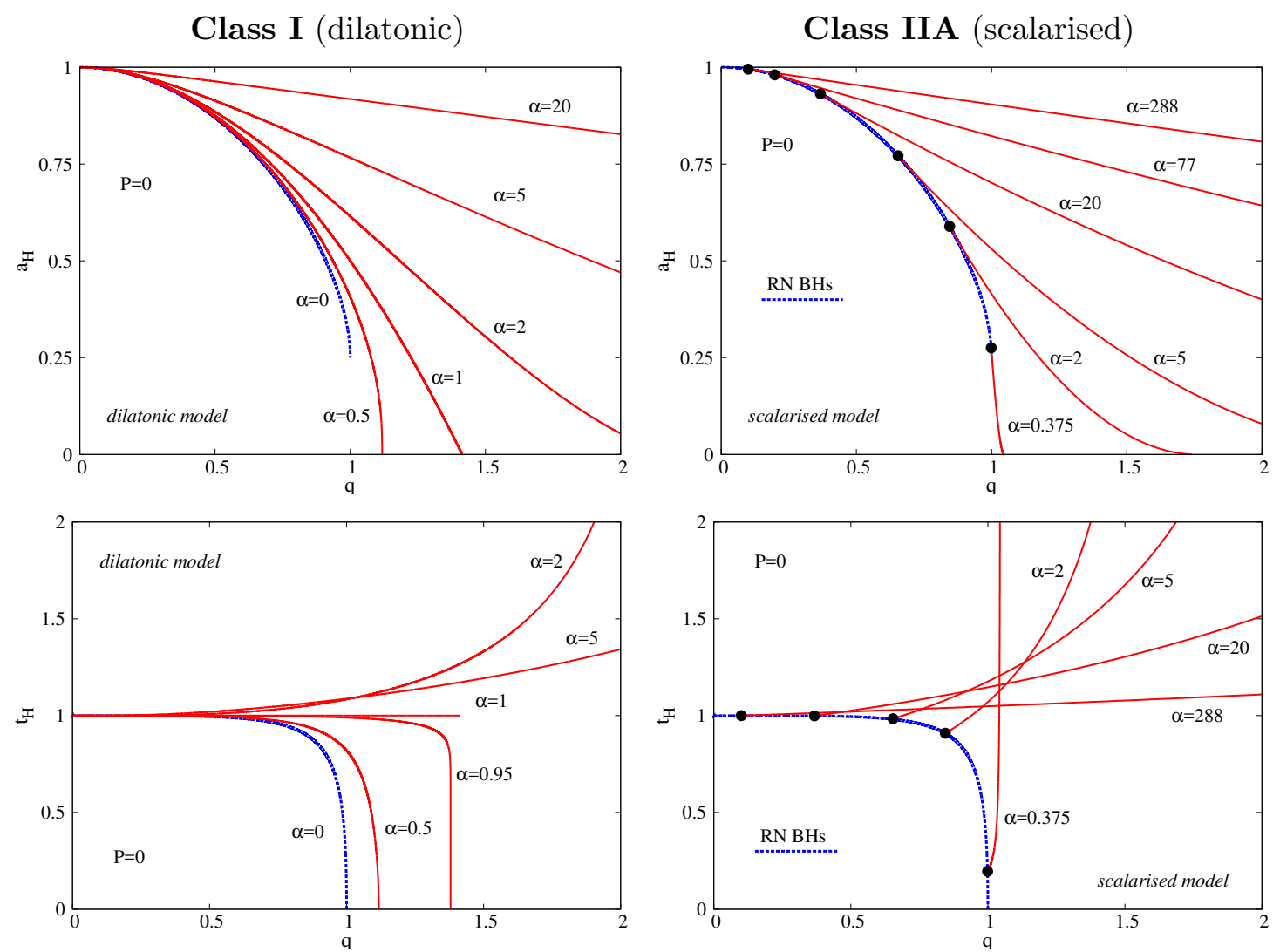

Figure 2. Reduced area $a_{H}$ (top panels) and reduced temperature $t_{H}$ (bottom panels) vs. reduced charge $q$ for dilatonic (left panels) and scalarised solutions (right panels). All solutions have $P=0$. The blue lines are the set of RN BHs $(\phi=0)$. The red lines are sequences of BHs with a nontrivial scalar field for a given $\alpha$. Different sequences are presented, for a range of values of $\alpha$. The black dots indicate the RN solutions from which the scalarised BHs bifurcate.

where the superscript ' $\mathrm{D}$ ' refers to dilatonic. The critical solution has, for any $\alpha>0$, a singular horizon, as one can see by evaluating the expression (3.12). The reduced temperature $t_{H}$, on the other hand, goes to zero for $\alpha<1$ and diverges for $\alpha>1$. The solutions with $\alpha=1$ have $t_{H}=1$. These features can be seen in figure 2 (left panels), where the behaviour of $a_{H}, t_{H}$ vs. $q$ are illustrated for several values of $\alpha$.

Let us now turn to our reference class IIA solutions. For the purely electric case these have been constructed in $[33,34]$. Let us briefly review their basic properties, emphasizing a comparison with class I solutions. Given a value of the coupling constant $\alpha>1 / 8$, the spherically symmetric scalarised BHs bifurcate from the corresponding $\mathrm{RN} \mathrm{BH}$, with a given $q=q(\alpha) \neq 0$, as discussed above. Keeping constant the parameter $\alpha$, this branch has a finite extent, ending again in a critical configuration. This limiting solution possess a singular horizon, as found when evaluating the Kretschmann scalar (3.12). The horizon area tends to zero as the critical solution is approached and the temperature diverges, while the mass and scalar charge remain finite. This behaviour parallels that of the dilatonic solutions with $\alpha>1$. In the region of the parameter space wherein scalarised and RN BHs 

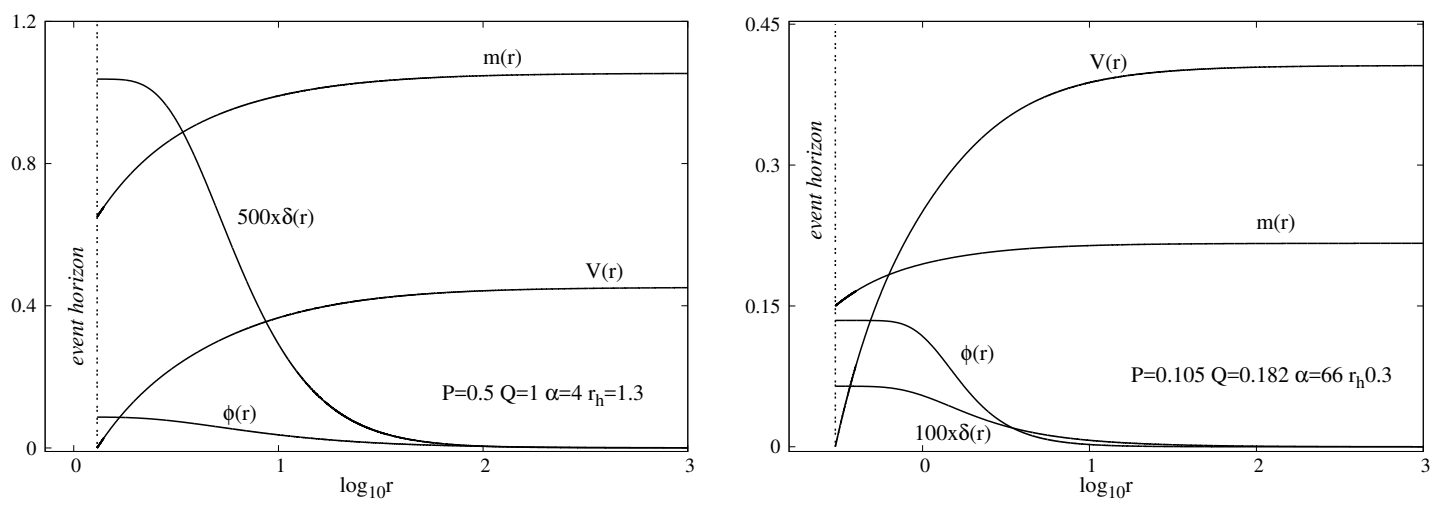

Figure 3. Examples of dyonic BHs radial profile functions for a dilatonic (left panel) and a scalarised (right panel) BH.

exist for the same $q$, one always finds that the scalarised solutions are entropically favoured over the RN BHs, as it is manifest from the top right panel of figure 2 .

The domain of existence of the purely electrically charged BHs of both types will be exhibited below in figure 5, where we also compare it with the dyonic case that we shall discuss next. This domain of existence, in an $(\alpha, q)$-diagram is bounded by two curves. In the dilatonic case, these curves correspond to the Schwarzschild $\mathrm{BH}$ and the line of critical solutions. In the scalarised case, these curves correspond to the aforementioned existence line - the set of RN solutions from which the scalarised BHs bifurcate - and, again, the line of critical solutions.

Finally, we remark that, for both dilatonic and scalarised solutions, along any branch with fixed $\alpha$, the ratio $q=\sqrt{Q^{2}+P^{2}} / M$ increases and becomes larger than unity at some stage. In this sense, overcharged $\mathrm{BHs}$ are possible, in contrast with the RN family.

\subsubsection{The dyonic BHs}

The purely electric solutions above, for both classes discussed, possess generalisations with a nonzero magnetic charge. The profile functions of illustrative dyonic BHs are shown in figure 3 , for both the dilatonic and scalarised cases.

Dyonic BHs preserve some, but not all, of the qualitative characteristics of the purely electric solutions. In the dilatonic case, the branch of solutions with a given $\alpha$ starts again from the Schwarzschild limiting solution (which has $a_{H}=1, t_{H}=1$ and $q=0$ ) and ends in a limiting configuration with $a_{H}>0, t_{H}=0$ and $q=q_{\max }>0$ - figure 4 (left panels). This limiting solution, however, is now an extremal BH (rather than a singular critical solution) and will be discussed in the next section.

Unlike the dilatonic solutions, which exist for arbitrarily small $q$ for any $\alpha$, scalarised BHs with a given $\alpha$ exist for $q>q_{\text {min }}$ only. They bifurcate from a RN BH (with $q>0$ ) and form a branch ending again on an extremal solution with vanishing horizon temperature and nonzero horizon area - figure 4 (right panels). As for purely electric solutions, for the same global charges $M, P, Q$, the scalarised solutions are entropically preferred over the corresponding RN solution. 

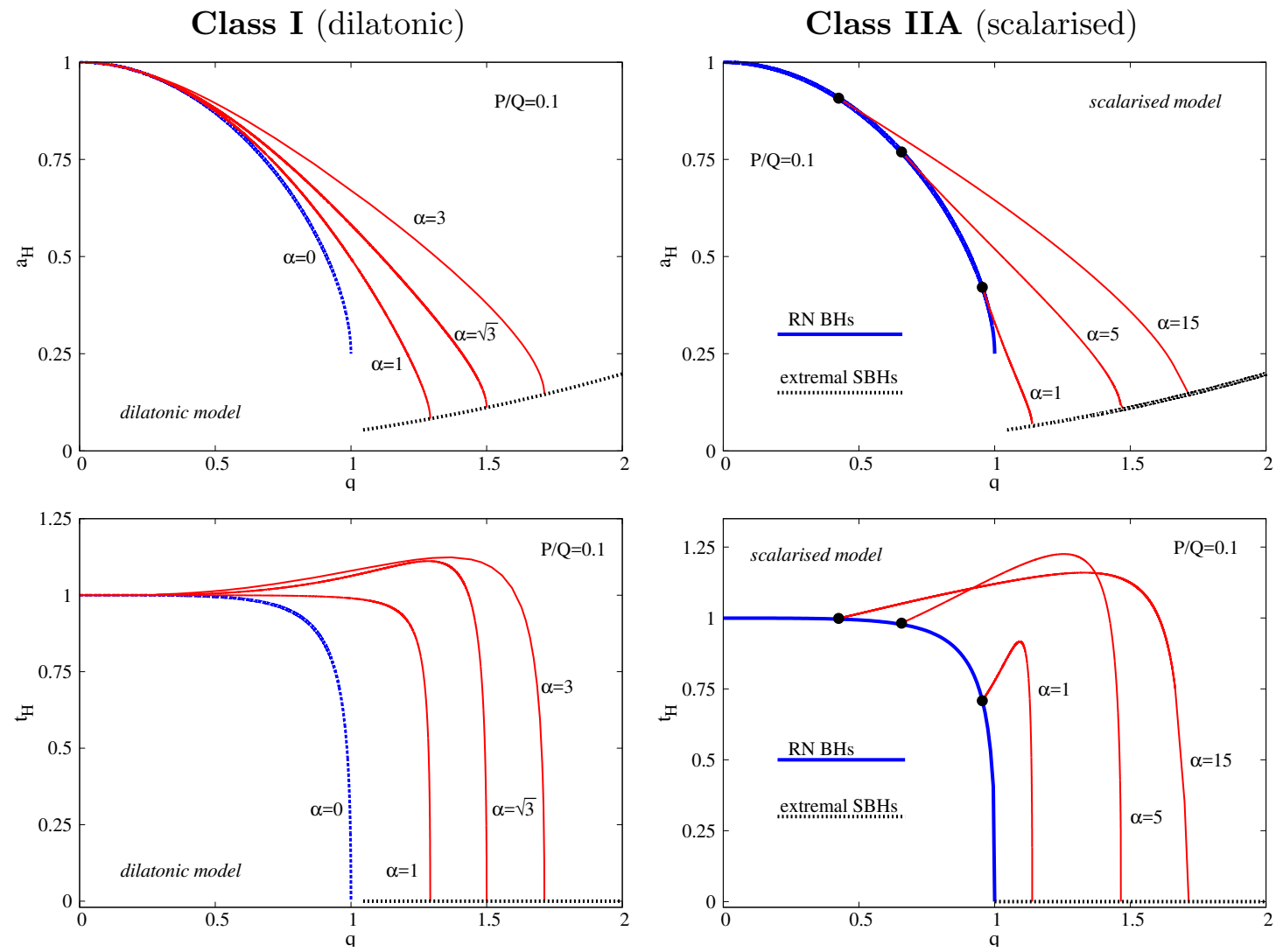

Figure 4. Same as figure 1 but now for dyonic BHs. The ratio between magnetic and electric charges is $P / Q=0.1$.

The domain of existence of the dyonic BHs is shown in figure 5 for several values of the ratio $P / Q$ and for both dilatonic and scalarised BHs. In particular, observe that in both cases, the maximal allowed value of $q$ for BHs with a given $\alpha$ decreases as the ratio $P / Q$ increases. In other words, the domain of existence shrinks, as the magnetic charge is increased, for fixed $Q$.

\section{Extremal BHs}

\subsection{Numerical construction}

To address extremal $\mathrm{BHs}$ one needs to impose a different near-horizon expansion to that in (3.9), which accounts for the degenerate horizon. The leading order terms of the appropriate expansion are:

$$
\begin{aligned}
N(r) & =N_{2}\left(r-r_{H}\right)^{2}+\ldots, & \delta(r) & =\delta_{0}+\delta_{1}\left(r-r_{H}\right)^{2 k-1}+\ldots, \\
\phi(r) & =\phi_{0}+\phi_{c}\left(r-r_{H}\right)^{k}+\ldots, & V(r) & =v_{1}\left(r-r_{H}\right)+\ldots .
\end{aligned}
$$

One can show that the next to leading order term in the expression of $N(r)$ is $\mathcal{O}\left(r-r_{H}\right)^{3}$. It is convenient to take $r_{H}$ and $\phi_{0}$ as essential parameters. Then the field equations imply

$$
Q=\frac{r_{H} \sqrt{f\left(\phi_{0}\right)}}{\sqrt{2}}, \quad P=\frac{r_{H}}{\sqrt{2 f\left(\phi_{0}\right)}}, \quad N_{2}=\frac{1}{r_{H}^{2}} .
$$



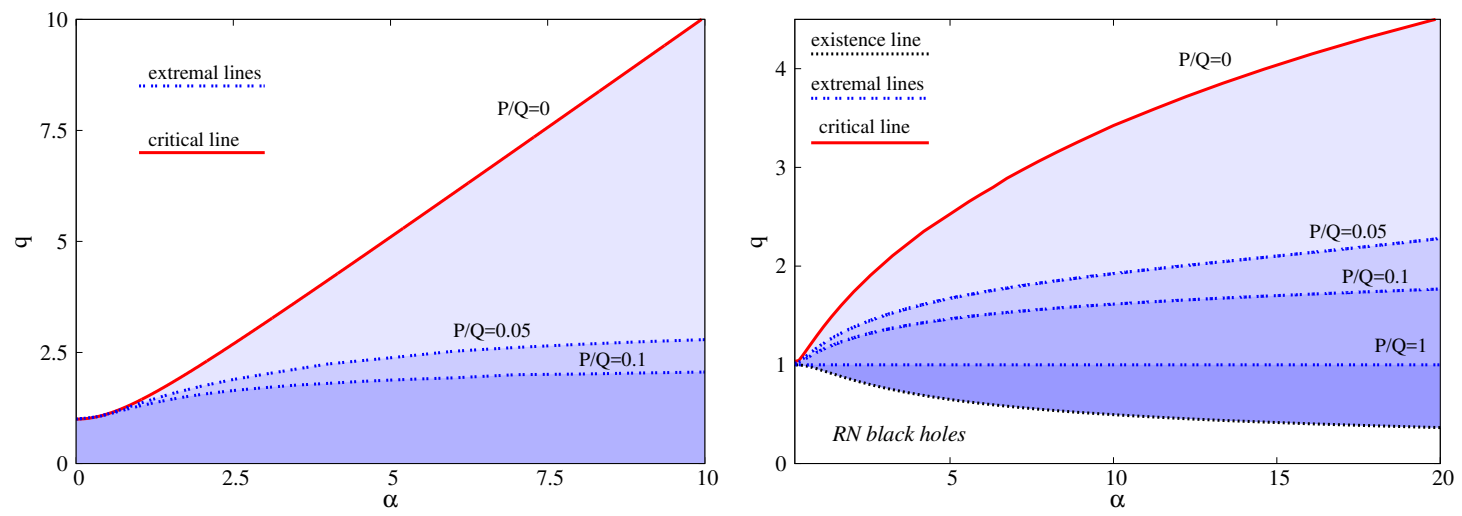

Figure 5. Domain of existence of dilatonic BHs (left panel) and scalarised BHs (right panel) for several values of the ratio $P / Q$.

Consequently, given an expression of the coupling function $f(\phi)$, one can express the value of the scalar field at the horizon $\phi_{0}$ as a function of $P, Q$, by solving the equation

$$
f\left(\phi_{0}\right)=\frac{Q}{P}, \quad \text { while } r_{H}=\sqrt{2 P Q} .
$$

The expansion (4.1) contains two free parameter $\phi_{c}$ and $\delta_{0}$ which are fixed by numerics, while $\delta_{1}, v_{1}$ are fixed as

$$
\delta_{1}=-\frac{r_{H} \phi_{c}^{2} k^{2}}{2 k-1}, \quad v_{1}=\frac{e^{-\delta_{0}} Q}{r_{H}^{2} f\left(\phi_{0}\right)} .
$$

The power $k$ in (4.1) is given by

$$
k=\frac{1}{2}\left(-1+\sqrt{1+2\left(\frac{f^{\prime}\left(\phi_{0}\right)}{f\left(\phi_{0}\right)}\right)^{2}}\right)>0,
$$

which, generically, takes non-integer values. A minimal requirement for the parameter $k$ comes from the condition that the function $\delta(r)$ is finite as $r \rightarrow r_{H}$, which implies

$$
k>1 / 2 \text {. }
$$

This assures that $g_{t t} \sim-\left(r-r_{H}\right)^{2}$, with the metric possessing an $A d S_{2} \times S^{2}$ near horizon geometry. Moreover, we have verified that both the Ricci and the Kretschmann scalars and the square of the Christoffel symbols are finite at the horizon (while they diverge for $k<1 / 2$ ).

A non-integer $k$, on the other hand, implies that a sufficiently higher order derivative of the curvature tensor will diverge as $r \rightarrow r_{H}$. Thus, for analytic solutions on the horizon (as extremal $\mathrm{RN}$ ), the power $k$ in the above near horizon expansion (4.1), (4.2) should be an integer. This imposes the stronger condition

$$
\frac{f^{\prime}\left(\phi_{0}\right)}{f\left(\phi_{0}\right)}= \pm \sqrt{2 p(p+1)}, \quad \text { with } p=1,2, \ldots
$$




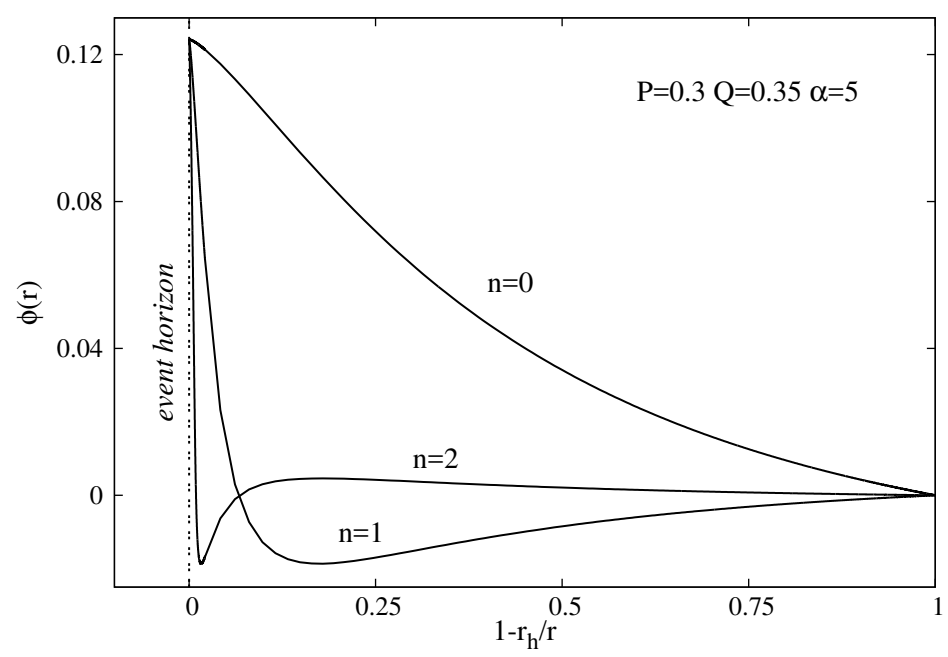

Figure 6. A sequence of scalar field profiles starting with the same horizon data in a scalarised model. Each solution possesses a different node number.

For the dilatonic case, condition (4.8) translates to [51, 52] (see also [53])

$$
\alpha=\sqrt{\frac{p(p+1)}{2}}
$$

again with an integer $p$. For scalarised solutions with the coupling function (2.18) the condition (4.8) becomes

$$
\alpha=\frac{p(p+1)}{4 \log \left(\frac{Q}{P}\right)} .
$$

The extremal solutions share the far field asymptotics (3.11) with the non-extremal ones; moreover, the relations (3.14)-(3.16) hold also for $T_{H}=0$.

We have constructed extremal solutions by using the same numerical approach as in the generic non-extremal case. The profile of the various functions resulting from the integration are not particularly enlightening, resembling those in the non-extremal case and shall not be shown here. But we would like to point out a peculiar feature of the extremal scalarised BHs. There exists a (presumably) infinite family of solutions with the same horizon data as specified by $\left(\phi_{0}, r_{H}\right)$ (or, equivalently, $(P, Q)$ ), labelled by their nodenumber $n$. This is illustrated in figure 6: the scalar field always starts at the same horizon value; however, the bulk profile is different. As expected for excited states, the mass of these solutions increases with $n$. We remark no excited configurations were found in the dilatonic case, which always has $n=0$.

\subsection{An analytic approach: the attractor mechanism and entropy function}

The numerical construction of the extremal BHs is a difficult numerical task. Let us now provide a different argument for the existence of the EMS extremal dyonic BHs: the existence of an exact solution describing a Robinson-Bertotti vacuum, namely an $A d S_{2} \times S^{2}$ 
spacetime. As for extremal RN BHs, we expect that this solution describes the neighbourhood of the event horizon of an extremal scalarised $\mathrm{BH}$ with nonzero magnetic and electric charges. As we shall see, both charges are mandatory for the Robinson-Bertotti vacuum to exist with a non-trivial scalar field.

To search for the Robinson-Bertotti vacuum we consider (2.5) with $a(r)^{2}=v_{0} r^{2}$, $b(r)^{2}=v_{1}, c(r)^{2}=v_{0} / r^{2}$, that is the line element

$$
d s^{2}=v_{0}\left(-r^{2} d t^{2}+\frac{d r^{2}}{r^{2}}\right)+v_{1}\left(d \theta^{2}+\sin ^{2} \theta d \varphi^{2}\right)
$$

and the matter fields ansatz

$$
A=e r d t+P \cos \theta d \varphi, \quad \phi=\phi_{0} .
$$

The constant parameters $\left\{v_{0}, v_{1} ; e, P, \phi_{0}\right\}$ satisfy a set of algebraic relations which result from the EMS equations (2.2)-(2.4). However, instead of attempting to solve these, we shall, in what follows, determine these parameters by using the formalism proposed in [4446], thus by extremizing an entropy function. This approach allows us also to compute the $\mathrm{BH}$ entropy and to show that the solutions exhibit an attractor-type behaviour.

Let us consider the Lagrangian density of the model, as read off from (2.1), evaluated for the ansatz (4.11)-(4.12) and integrated over the angular coordinates,

$$
\mathcal{L}=\frac{1}{16 \pi} \int d \theta d \varphi \sqrt{-g}\left(R-2(\nabla \phi)^{2}-f(\phi) F^{2}\right)=\frac{1}{2}\left[v_{0}-v_{1}+f\left(\phi_{0}\right)\left(\frac{e^{2} v_{1}}{v_{0}}-\frac{P^{2} v_{0}}{v_{1}}\right)\right] .
$$

Then, following [44-46], we define the entropy function $\mathcal{E}$ by taking the Legendre transform of the above integral with respect to a parameter $Q$,

$$
\mathcal{E}=2 \pi(e Q-\mathcal{L}),
$$

where $Q=\partial \mathcal{E} / \partial e$ is the electric charge of the solutions. It follows as a consequence of the equations of motion that the constants $\left\{v_{0}, v_{1} ; e, \phi_{0}\right\}$ are solutions of the equations

$$
\frac{\partial \mathcal{E}}{\partial \phi_{0}}=0, \quad \frac{\partial \mathcal{E}}{\partial v_{i}}=0, \quad \frac{\partial \mathcal{E}}{\partial e}=0,
$$

or, explicitly,

$$
\begin{aligned}
\frac{\partial \mathcal{E}}{\partial v_{0}}=0 & \Rightarrow-1+\left(\frac{v_{1}}{v_{0}^{2}} e^{2}+\frac{1}{v_{1}} P^{2}\right) f\left(\phi_{0}\right)=0, \\
\frac{\partial \mathcal{E}}{\partial v_{1}}=0 & \Rightarrow 1-\left(\frac{1}{v_{0}} e^{2}+\frac{v_{0}}{v_{1}^{2}} P^{2}\right) f\left(\phi_{0}\right)=0, \\
\frac{\partial \mathcal{E}}{\partial \phi_{0}}=0 & \Rightarrow\left(P^{2} v_{0}^{2}-e^{2} v_{1}^{2}\right) \frac{d f}{d \phi_{0}}=0, \\
\frac{\partial \mathcal{E}}{\partial e}=0 & \Rightarrow Q=e \frac{v_{1}}{v_{0}} f\left(\phi_{0}\right) .
\end{aligned}
$$

The sum of (4.16) and (4.17) leads to the generic relation

$$
v_{0}=v_{1} .
$$


Thus, the 'radius' of the $A d S_{2}$ is always equal with the one of $S^{2}$ in the metric (4.11). Then, the equation (4.19) becomes

$$
Q=e f\left(\phi_{0}\right) .
$$

Consequently, eq. (4.18) implies the existence of two different families of solutions:

(a) Eq. (4.18) is solved if the coupling function obeys $d f / d \phi_{0}=0$. Then, $e$ and $P$ are independent quantities and, from (4.16),

$$
v_{0}=v_{1}=\left(e^{2}+P^{2}\right) f\left(\phi_{0}\right) .
$$

This family of solutions is only possible in the scalarised case. In this case, $d f / d \phi_{0}=0$, with $\phi_{0}=0$. Therefore, one obtains the near horizon geometry of the extremal RN $\mathrm{BH}$, with a vanishing scalar field.

(b) Eq. (4.18) is also solved if

$$
e=P, \quad \stackrel{(4.21)}{\Rightarrow} \quad Q=\operatorname{Pf}\left(\phi_{0}\right),
$$

and, from (4.16),

$$
v_{0}=v_{1}=2 P^{2} f\left(\phi_{0}\right) .
$$

This family of solutions is possible for both the scalarised and dilatonic cases and demands both $Q, P$ to be non-vanishing.

The scalarisation mechanism is encoded in the existence of two different types of attractor solutions in the scalarised EMS models. This contrasts with the case of the dilatonic coupling, for which condition (4.23) is mandatory and only one type of solutions exists, that requires both electric and magnetic charges to be present.

It is straighforward to check that in both cases the entropy function, $\mathcal{E}$, evaluated at the attractor critical point is given by one-quarter of the area of angular sector in (4.11),

$$
S=\pi v_{1} .
$$

Finally, we remark that the correspondence of the above parameters with the ones in the near horizon expansion of the extremal BHs in section 4.1 is straightforward:

$$
v_{1}=r_{H}^{2}, \quad v_{0}=1 / N_{2} .
$$

\section{Discussion}

In this paper we have investigated the properties of static electromagnetically charged BHs with a non-trivial scalar field profile in EMS models, which are described by (2.1). A natural classification of these EMS models arises from the standard RN BH of electrovacuum being, or not, a solution. This divides EMS models into two classes. Class I, or dilatonic-type, 
does not admit RN as a solution. We have illustrated this class by a well-known family of dilatonic BHs that naturally emerge in the low energy limit of string theory, as well as in Kaluza-Klein theories. Class II, or scalarised-type, admits RN as a solution. The RN BH may, or may not, be continuously connected to the new BHs with a scalar field profile, naturally leading to two subclasses. In class IIA RN is continuously connected to the new BHs. This class contains the models wherein spontaneous scalarisation of the RN $\mathrm{BH}$ occurs [33], dynamically leading to the new scalarised BHs. We have illustrated this class by a particular choice of coupling function, introduced in [33] in this context. In class IIB, RN is not continously connected to the new BHs and the RN BH is not unstable against scalarisation.

One of the motivations for this work was to understand the effect of a magnetic charge in the EMS BHs. In the well known dilatonic case, dyonic BHs have a regular extremal limit, whereas purely electrically (or magnetically) charged ones do not; the latter become singular, approaching a critical solution when endowed with the maximal possible charge for a given mass. Given the special features of smooth extremal solutions, it is of interest to understand the status of these solutions in the generic EMS case, since for purely electic scalarised BHs maximal charge led to critical, rather than extremal, solutions [33, 34]. Here we have shown that for scalarised BHs the conclusion is similar to dilatonic BHs (within a certain coupling regime) in this respect: dyonic BHs can have a regular extremal limit. Our analysis also allows constructing such dyonic extremal solutions for arbitrary coupling in the dilatonic case, since solutions where only known (in analytic closed form) for some particular values of the coupling. Morover, despite the defining difference in the two classes of solutions, figure 2 and 4 show that these two classes, for the illustrative families, present similar trends in the behaviour of physical quantities.

As evidence for the existence of dyonic extremal scalarised BHs, we have made use of the fact one expects such solutions to have a near-horizon geometry which is, itself, a solution of the field equations. Both for RN and Kerr extremal BHs (when $T_{H}=0$ ), the near horizon geometry has an enhanced symmetry that contains an $A d S_{2}$ geometry (for Kerr, there exists a non-trivial fibration of an $S^{1}$ on $A d S_{2}$ in the near horizon geometry). It was proven in $[44,45]$ that the existence of $A d S_{2}$ factor is, in fact, at the basis of the attractor mechanism for extremal BHs rather than supersymmetry [26, 27]. In string theory, the attractor mechanism provides a non-renormalization theorem for the matching of statistical and thermodynamic entropies of extremal BHs [54] (see, also, section 5 of [55]). Here, the attractor mechanism provides a clear and simple explanation of why the extremal limit is a naked singularity for solutions with a single charge and a smooth geometry for dyonic BHs. Besides enabling a partial analytical understanding of the extremal solutions, analysing the near horizon geometry provides an insight on how scalarisation leaves a trace at the level of attractors, allowing two families of near horizon geometries.

Let us close considering some future research. It would be interesting to motivate class II models from a more fundamental viewpoint. In this respect, we remark that (2.1) may be viewed as a member of a more general family of low energy string theory actions (see, e.g., [56]). For example, in four dimensions, the effective string theory can be described by $\mathcal{N}=2$ supergravity (and its deformations [57]), with the generic bosonic Lagrangian 
density

$$
\begin{aligned}
L= & -\frac{R}{2}+h_{i \bar{\jmath}} \partial_{\mu} z^{i} \partial^{\mu} \bar{z}^{\bar{\jmath}}+\frac{1}{4} \mathcal{F}_{\Lambda \Sigma}(z, \bar{z}) F_{\mu \nu}^{\Lambda} F^{\Sigma \mu \nu} \\
& +\frac{1}{8 e_{D}} \mathcal{R}_{\Lambda \Sigma}(z, \bar{z}) \epsilon^{\mu \nu \rho \sigma} F_{\mu \nu}^{\Lambda} F_{\rho \sigma}^{\Sigma}-V(z, \bar{z}) .
\end{aligned}
$$

The model possesses $n_{s}$ complex scalars $z^{i}, i=1, \ldots, n_{s}$, coupled to the vector fields $F_{\mu \nu}^{\Lambda}$ in a non-minimal way through the real symmetric matrices $\mathcal{F}_{\Lambda \Sigma}(z, \bar{z}), \mathcal{R}_{\Lambda \Sigma}(z, \bar{z})$ and span a special Kähler manifold with the metric $h_{i \bar{\jmath}}$. The scalar potential $V(z, \bar{z})$ originates from electric-magnetic Fayet-Illiopulos terms; a consistent truncation with only one scalar field and a concrete potential was presented in [58] (see, also, [59-61]). Despite the existence of a potential for the scalar field, one can consider a situation when the effective cosmological constant vanishes at the boundary even if the self-interaction in the bulk does not [62] - exact asymptotically flat hairy black hole solutions with a non-trivial dilaton potential were obtained in [19]. We observe that the moduli metric and coupling with the gauge field can be non-trivial. Depending of their form, the RN BH may, or may not, be obtained as a solution of the theory. For the dilatonic coupling (2.12) and a trivial moduli metric, the limit $\phi \rightarrow 0$ does not provide a consistent truncation and so RN BH is not a solution of the theory. However, this is not necessarily the case for any metric $h_{i \bar{\jmath}}$. Finding a concrete realisation of class II models in this context would be very promising.

Amongst the several extension of the discussion herein, including in (2.1) an axiontype term could be interesting, due to the high energy physics motivation for axions. Another obvious extension would be the consideration of solutions with less symmetry, either rotating solutions or solutions connected to zero modes with $\ell \neq 0$.

Finally, as a speculation, one can notice some analogy of the scalarised BHs and the AdS holographic duals of superconductors (the $s$-wave case) [63]. The general mechanism appears to be the following: for both asymptotics, the RN BH remains a solution of the full model. However, for some range of the parameters, the non-trivial coupling of the scalar field with the Maxwell field gives a tachyonic mass for the vacuum scalar perturbations around the RN BH, with the appearance of a scalar condensate. This implies the occurrence of a branch of scalarised BHs, which are generically thermodynamically favoured over the $\mathrm{RN}$ configurations. It would be interesting to further pursue this apparent parallelism in the Minkowskian case, and to investigate the possible relevance of these aspects in providing 'dual' descriptions to phenomena observed in condensed matter physics.

\section{Acknowledgments}

The work of D.A. has been funded by the Fondecyt Regular Grant 1161418. The work of C.H., A.P., and E.R. is supported by the Fundação para a Ciência e a Tecnologia (FCT) project UID/MAT/04106/2019 (CIDMA), by CENTRA (FCT) project UID/FIS/00099/2013, by national funds (OE), through FCT, I.P., in the scope of the framework contract foreseen in the numbers 4,5 and 6 of the article 23, of the Decree-Law $57 / 2016$, of August 29, changed by Law 57/2017, of July 19. We acknowledge support 
from the project PTDC/FIS-OUT/28407/2017. This work has also been supported by the European Union's Horizon 2020 research and innovation (RISE) programmes H2020MSCA-RISE-2015 Grant No. StronGrHEP-690904 and H2020-MSCA-RISE-2017 Grant No. FunFiCO-777740. A. P. is supported by the FCT grant PD/BD/142842/2018. E. R. gratefully acknowledges the support of the Alexander von Humboldt Foundation. The authors would like to acknowledge networking support by the COST Action CA16104.

\section{A Exact solutions with a linear coupling}

\section{A.1 Purely electric BHs}

Purely electric dilatonic solutions of (2.1) with the dilatonic coupling (2.12) where first considered by Gibbons and Maeda [16] and Garfinkle, Horowitz and Strominger [17]. The BH solution has the line element (2.5) with

$$
a(r)^{2}=\frac{1}{c(r)^{2}}=\left(1-\frac{r_{+}}{r}\right)\left(1-\frac{r_{-}}{r}\right)^{\frac{1-\alpha^{2}}{1+\alpha^{2}}}, \quad b(r)=r\left(1-\frac{r_{-}}{r}\right)^{\frac{\alpha^{2}}{1+\alpha^{2}}},
$$

together with the Maxwell potential and dilaton field ${ }^{5}$

$$
A=\frac{Q}{r} d t, \quad e^{2 \phi}=\left(1-\frac{r_{-}}{r}\right)^{\frac{2 \alpha}{1+\alpha^{2}}} .
$$

The two free parameters $r_{+}, r_{-}$(with $r_{-}<r_{+}$) are related to the ADM mass, $M$, and (total) electric charge, $Q$, by

$$
M=\frac{1}{2}\left[r_{+}+\left(\frac{1-\alpha^{2}}{1+\alpha^{2}}\right) r_{-}\right], \quad Q=\left(\frac{r_{-} r_{+}}{1+\alpha^{2}}\right)^{\frac{1}{2}} .
$$

For all $\alpha$, the surface $r=r_{H}=r_{+}$is the location of the (outer) event horizon, with

$$
A_{H}=4 \pi r_{+}^{2}\left(1-\frac{r_{-}}{r_{+}}\right)^{\frac{2 \alpha^{2}}{1+\alpha^{2}}}, \quad T_{H}=\frac{1}{4 \pi} \frac{1}{r_{+}-r_{-}}\left(1-\frac{r_{-}}{r_{+}}\right)^{\frac{2}{1+\alpha^{2}}} .
$$

The extremal limit, which corresponds to the coincidence limit $r_{-}=r_{+}$, results in a singular solution (as can be seen e.g. by evaluating the Kretschmann scalar). In this limit, the area of the event horizon goes to zero for $\alpha \neq 0$. The Hawking temperature, however, only goes to zero in the extremal limit for $\alpha<1$, while for $\alpha=1$ it approaches a constant, and for $\alpha>1$ it diverges.

The reduced quantities (3.19) have the following exact expressions:

$$
\begin{aligned}
q & =\frac{2 \sqrt{\left(1+\alpha^{2}\right) x}}{1+\alpha^{2}(1-x)+x}, \quad a_{H}=\frac{\left(1+\alpha^{2}\right)^{2}(1-x)^{\frac{2 \alpha^{2}}{1+\alpha^{2}}}}{\left(1+\alpha^{2}(1-x)+x\right)^{2}}, \\
t_{H} & =\frac{(1-x)^{\frac{1-\alpha^{2}}{1+\alpha^{2}}}\left(1+\alpha^{2}(1-x)+x\right)}{1+\alpha^{2}},
\end{aligned}
$$

where $0 \leqslant x \leqslant 1$ is a parameter.

\footnotetext{
${ }^{5}$ Following the conventions in the work, we fix $\phi(\infty)=0$ for all solutions in the appendix.
} 


\section{A.2 Dyonic BHs}

\section{A.2.1 $\alpha=1$}

A dyonic dilatonic BH solution of (2.1), with the dilatonic coupling (2.12) and $\alpha=1$, was found in [43], and extensively discussed in the literature, since it can be embedded in $\mathcal{N}=4$ supergravity. Taking the form (2.5), it has

$$
\phi=\frac{1}{2} \log \frac{(r+\Sigma)}{(r-\Sigma)}, \quad a(r)^{2}=\frac{1}{c(r)^{2}}=\frac{\left(r-r_{+}\right)\left(r-r_{-}\right)}{\left(r^{2}-\Sigma^{2}\right)}, \quad b(r)^{2}=r^{2}-\Sigma^{2},
$$

where

$$
r_{ \pm}=M \pm \sqrt{M^{2}+\Sigma^{2}-Q^{2}-P^{2}}
$$

and the outer horizon is at $r_{H}=r_{+}$, while $M, Q, P$ are the mass and electric and magnetic charges. $\Sigma$ corresponds to the scalar charge, which, however, is not an independent parameter (the hair is secondary):

$$
\Sigma=\frac{P^{2}-Q^{2}}{2 M}
$$

The extremal limit of the above solution corresponds to $r_{+}=r_{-}$, in which case one finds two relations between the charges

$$
0=M^{2}+\Sigma^{2}-Q^{2}-P^{2} \Longrightarrow(M+\Sigma)^{2}-2 P^{2}=0 \quad \text { and } \quad(M-\Sigma)^{2}-2 Q^{2}=0 .
$$

The horizon area and Hawking temperature of the solutions are

$$
A_{H}=4 \pi\left(2 M r_{+}-P^{2}-Q^{2}\right), \quad T_{H}=\frac{1}{2 \pi} \frac{r_{+}-M}{2 M r_{+}-P^{2}-Q^{2}} .
$$

The expression of the reduced quantities is more involved in this case:

$$
a_{H}=\frac{1}{4}\left(2 x-q^{2}\right), \quad t_{H}=\frac{4(x-1)}{2 x-q^{2}},
$$

with $x$ a parameter expressed in terms of $q$ as a solution of the equation (where $k=\frac{P}{Q}$ )

$$
q^{4}-\frac{4\left(1+k^{2}\right)^{2}}{\left(1-k^{2}\right)^{2}}\left(q^{2}+x(x-2)\right)=0
$$

\section{A.2.2 $\alpha=\sqrt{3}$}

A dyonic dilatonic BH solution of (2.1), with the dilatonic coupling (2.12) and $\alpha=\sqrt{3}$, was found in [41, 42]. This case arises from a suitable Kaluza-Klein reduction of a fivedimensional vacuum $\mathrm{BH}$. In the extremal limit, one obtains a non-BPS BH that can be embedded in $\mathcal{N}=2$ supergravity.

The generic solution can be written again in the form (2.5) with

$$
a(r)^{2}=\frac{1}{c(r)^{2}}=\frac{\left(r-r_{+}\right)\left(r-r_{-}\right)}{\sqrt{A B}}, \quad b(r)^{2}=\sqrt{A B} \quad \text { and } \quad e^{4 \phi(r) / \sqrt{3}}=\frac{A}{B},
$$


where

$$
A=\left(r-r_{A_{+}}\right)\left(r-r_{A_{-}}\right), \quad B=\left(r-r_{B_{+}}\right)\left(r-r_{B_{-}}\right) .
$$

In the above relations one defines

$$
r_{ \pm}=M \pm \sqrt{M^{2}+\Sigma^{2}-P^{2}-Q^{2}},
$$

where, again, the outer horizon is at $r_{H}=r_{+}$, and

$$
r_{A_{ \pm}}=\frac{1}{\sqrt{3}} \Sigma \pm P \sqrt{\frac{2 \Sigma}{\Sigma-\sqrt{3} M}}, \quad r_{B_{ \pm}}=-\frac{1}{\sqrt{3}} \Sigma \pm Q \sqrt{\frac{2 \Sigma}{\Sigma+\sqrt{3} M}} .
$$

The solution possesses again three parameters $M, Q, P$ which fix the scalar charge $\Sigma$ via the equation

$$
\frac{2}{\sqrt{3}} \Sigma=\frac{Q^{2}}{\sqrt{3} M+\Sigma}-\frac{P^{2}}{\sqrt{3} M-\Sigma},
$$

while the horizon area and the Hawking temperature are given by

$$
\begin{aligned}
& A_{H}=4 \pi \sqrt{\left(r_{+}-r_{A_{+}}\right)\left(r_{+}-r_{A_{-}}\right)\left(r_{+}-r_{B_{+}}\right)\left(r_{+}-r_{B_{-}}\right)}, \\
& T_{H}=\frac{1}{4 \pi} \frac{r_{+}-r_{-}}{\sqrt{\left(r_{+}-r_{A_{+}}\right)\left(r_{+}-r_{A_{-}}\right)\left(r_{+}-r_{B_{+}}\right)\left(r_{+}-r_{B_{-}}\right)}} .
\end{aligned}
$$

The corresponding expressions for $a_{H}$ and $t_{H}$ as a function of $q$ (and the ratio $P / Q$ ) can be derived directly from the above relations; however, they are too complicated to include here.

Open Access. This article is distributed under the terms of the Creative Commons Attribution License (CC-BY 4.0), which permits any use, distribution and reproduction in any medium, provided the original author(s) and source are credited.

\section{References}

[1] V. Moncrief, Odd-parity stability of a Reissner-Nordstrom black hole, Phys. Rev. D 9 (1974) 2707 [INSPIRE].

[2] V. Moncrief, Stability of Reissner-Nordstrom black holes, Phys. Rev. D 10 (1974) 1057 [INSPIRE].

[3] G.W. Gibbons and C.M. Hull, A Bogomolny bound for general relativity and solitons in $N=2$ supergravity, Phys. Lett. B 109 (1982) 190.

[4] G.W. Gibbons and P.K. Townsend, Vacuum interpolation in supergravity via super p-branes, Phys. Rev. Lett. 71 (1993) 3754 [hep-th/9307049] [INSPIRE].

[5] I. Robinson, A solution of the Maxwell-Einstein equations, Bull. Acad. Pol. Sci. Ser. Sci. Math. Astron. Phys. 7 (1959) 351 [inSPIRE].

[6] B. Bertotti, Uniform electromagnetic field in the theory of general relativity, Phys. Rev. 116 (1959) 1331 [INSPIRE]. 
[7] S.D. Majumdar, A class of exact solutions of Einstein's field equations, Phys. Rev. 72 (1947) 390 [INSPIRE].

[8] A. Papapetrou, Einstein's theory of gravitation and flat space, Proc. Roy. Irish Acad. 52A (1948) 11 [INSPIRE].

[9] J.B. Hartle and S.W. Hawking, Solutions of the Einstein-Maxwell equations with many black holes, Commun. Math. Phys. 26 (1972) 87 [InSPIRE].

[10] A.E. Mayo and J.D. Bekenstein, No hair for spherical black holes: charged and nonminimally coupled scalar field with selfinteraction, Phys. Rev. D 54 (1996) 5059 [gr-qc/9602057] [INSPIRE].

[11] T. Kaluza, Zum Unitätsproblem der Physik, Sitzungsber. Preuss. Akad. Wiss. Berlin (Math. Phys. ) 1921 (1921) 966 [Int. J. Mod. Phys. D 27 (2018) 1870001] [arXiv:1803.08616] [INSPIRE].

[12] O. Klein, Quantum theory and five-dimensional theory of relativity (in German and English), Z. Phys. 37 (1926) 895 [Surv. High Energ. Phys. 5 (1986) 241] [InSPIRE].

[13] A. Einstein, The field equations of gravitation, Sitzungsber. Preuss. Akad. Wiss. Berlin 1915 (1915) 844 [INSPIRE].

[14] T. Appelquist, A. Chodos and P.G.O. Freund, Modern Kaluza-Klein theories, Addison Wesley, Reading, U.S.A. (1987).

[15] E. Cremmer and B. Julia, The $N=8$ supergravity theory. 1. The lagrangian, Phys. Lett. 80B (1978) 48 [INSPIRE].

[16] G.W. Gibbons and K.-i. Maeda, Black holes and membranes in higher dimensional theories with dilaton fields, Nucl. Phys. B 298 (1988) 741 [INSPIRE].

[17] D. Garfinkle, G.T. Horowitz and A. Strominger, Charged black holes in string theory, Phys. Rev. D 43 (1991) 3140 [Erratum ibid. D 45 (1992) 3888] [INSPIRE].

[18] J.F.M. Delgado, C.A.R. Herdeiro and E. Radu, Violations of the Kerr and Reissner-Nordström bounds: horizon versus asymptotic quantities, Phys. Rev. D 94 (2016) 024006 [arXiv: 1606.07900] [INSPIRE].

[19] A. Anabalon, D. Astefanesei and R. Mann, Exact asymptotically flat charged hairy black holes with a dilaton potential, JHEP 10 (2013) 184 [arXiv:1308.1693] [INSPIRE].

[20] D. Astefanesei, D. Choque, F. Gómez and R. Rojas, Thermodynamically stable asymptotically flat hairy black holes with a dilaton potential, JHEP 03 (2019) 205 [arXiv: 1901.01269] [INSPIRE].

[21] A. Anabalón and D. Astefanesei, On attractor mechanism of AdS 4 black holes, Phys. Lett. B 727 (2013) 568 [arXiv: 1309.5863] [INSPIRE].

[22] G.W. Gibbons, Antigravitating black hole solitons with scalar hair in $N=4$ supergravity, Nucl. Phys. B 207 (1982) 337 [inSPIRE].

[23] C.A.R. Herdeiro and E. Radu, Asymptotically flat black holes with scalar hair: a review, Int. J. Mod. Phys. D 24 (2015) 1542014 [arXiv: 1504.08209] [INSPIRE].

[24] G.W. Gibbons, R. Kallosh and B. Kol, Moduli, scalar charges and the first law of black hole thermodynamics, Phys. Rev. Lett. 77 (1996) 4992 [hep-th/9607108] [INSPIRE].

[25] D. Astefanesei, R. Ballesteros, D. Choque and R. Rojas, Scalar charges and the first law of black hole thermodynamics, Phys. Lett. B 782 (2018) 47 [arXiv:1803.11317] [INSPIRE]. 
[26] S. Ferrara, R. Kallosh and A. Strominger, $N=2$ extremal black holes, Phys. Rev. D 52 (1995) R5412 [hep-th/9508072] [INSPIRE].

[27] S. Ferrara and R. Kallosh, Supersymmetry and attractors, Phys. Rev. D 54 (1996) 1514 [hep-th/9602136] [INSPIRE].

[28] R. Kallosh, N. Sivanandam and M. Soroush, The non-BPS black hole attractor equation, JHEP 03 (2006) 060 [hep-th/0602005] [INSPIRE].

[29] F. Larsen, A nAttractor mechanism for nAdS $S_{2} / n C F T_{1}$ holography, JHEP 04 (2019) 055 [arXiv: 1806.06330] [INSPIRE].

[30] H.W. Lin, J. Maldacena and Y. Zhao, Symmetries near the horizon, JHEP 08 (2019) 049 [arXiv: 1904.12820] [INSPIRE].

[31] J. Martin and J. Yokoyama, Generation of large-scale magnetic fields in single-field inflation, JCAP 01 (2008) 025 [arXiv:0711.4307] [INSPIRE].

[32] A. Maleknejad, M.M. Sheikh-Jabbari and J. Soda, Gauge fields and inflation, Phys. Rept. 528 (2013) 161 [arXiv: 1212.2921] [INSPIRE].

[33] C.A.R. Herdeiro, E. Radu, N. Sanchis-Gual and J.A. Font, Spontaneous scalarization of charged black holes, Phys. Rev. Lett. 121 (2018) 101102 [arXiv: 1806.05190] [INSPIRE].

[34] P.G.S. Fernandes, C.A.R. Herdeiro, A.M. Pombo, E. Radu and N. Sanchis-Gual, Spontaneous scalarisation of charged black holes: coupling dependence and dynamical features, Class. Quant. Grav. 36 (2019) 134002 [arXiv: 1902.05079] [INSPIRE].

[35] S.S. Gubser, Phase transitions near black hole horizons, Class. Quant. Grav. 22 (2005) 5121 [hep-th/0505189] [INSPIRE].

[36] I.Z. Stefanov, S.S. Yazadjiev and M.D. Todorov, Phases of $4 D$ scalar-tensor black holes coupled to Born-Infeld nonlinear electrodynamics, Mod. Phys. Lett. A 23 (2008) 2915 [arXiv:0708.4141] [INSPIRE].

[37] D.D. Doneva, S.S. Yazadjiev, K.D. Kokkotas and I.Z. Stefanov, Quasi-normal modes, bifurcations and non-uniqueness of charged scalar-tensor black holes, Phys. Rev. D 82 (2010) 064030 [arXiv: 1007.1767] [INSPIRE].

[38] Y.S. Myung and D.-C. Zou, Instability of Reissner-Nordström black hole in Einstein-Maxwell-scalar theory, Eur. Phys. J. C 79 (2019) 273 [arXiv:1808.02609] [INSPIRE].

[39] Y.S. Myung and D.-C. Zou, Quasinormal modes of scalarized black holes in the Einstein-Maxwell-scalar theory, Phys. Lett. B 790 (2019) 400 [arXiv:1812.03604] [INSPIRE].

[40] Y.S. Myung and D.-C. Zou, Stability of scalarized charged black holes in the Einstein-Maxwell-scalar theory, Eur. Phys. J. C 79 (2019) 641 [arXiv:1904.09864] [INSPIRE].

[41] P. Dobiasch and D. Maison, Stationary, spherically symmetric solutions of Jordan's unified theory of gravity and electromagnetism, Gen. Rel. Grav. 14 (1982) 231 [InSPIRE].

[42] G.W. Gibbons and D.L. Wiltshire, Black holes in Kaluza-Klein theory, Annals Phys. 167 (1986) 201 [Erratum ibid. 176 (1987) 393] [INSPIRE].

[43] R. Kallosh et al., Supersymmetry as a cosmic censor, Phys. Rev. D 46 (1992) 5278 [hep-th/9205027] [INSPIRE]. 
[44] A. Sen, Black hole entropy function and the attractor mechanism in higher derivative gravity, JHEP 09 (2005) 038 [hep-th/0506177] [INSPIRE].

[45] D. Astefanesei et al., Rotating attractors, JHEP 10 (2006) 058 [hep-th/0606244] [INSPIRE].

[46] A. Sen, Black hole entropy function, attractors and precision counting of microstates, Gen. Rel. Grav. 40 (2008) 2249 [arXiv:0708.1270] [InSPIRE].

[47] Z.-Y. Fan and H. Lü, Charged black holes with scalar hair, JHEP 09 (2015) 060 [arXiv: 1507.04369] [INSPIRE].

[48] C.W. Misner and D.H. Sharp, Relativistic equations for adiabatic, spherically symmetric gravitational collapse, Phys. Rev. 136 (1964) B571 [INSPIRE].

[49] L. Smarr, Mass formula for Kerr black holes, Phys. Rev. Lett. 30 (1973) 71 [Erratum ibid. 30 (1973) 521] [INSPIRE].

[50] G.H. Derrick, Comments on nonlinear wave equations as models for elementary particles, J. Math. Phys. 5 (1964) 1252 [InSPIRE].

[51] D. Gal'tsov, M. Khramtsov and D. Orlov, "Triangular" extremal dilatonic dyons, Phys. Lett. B 743 (2015) 87 [arXiv: 1412.7709] [INSPIRE].

[52] A. Zadora, D.V. Gal'tsov and C.-M. Chen, Higher-n triangular dilatonic black holes, Phys. Lett. B 779 (2018) 249 [arXiv:1712.06570] [INSPIRE].

[53] M.E. Abishev, K.A. Boshkayev, V.D. Dzhunushaliev and V.D. Ivashchuk, Dilatonic dyon black hole solutions, Class. Quant. Grav. 32 (2015) 165010 [arXiv:1504.07657] [INSPIRE].

[54] A. Dabholkar, A. Sen and S.P. Trivedi, Black hole microstates and attractor without supersymmetry, JHEP 01 (2007) 096 [hep-th/0611143] [INSPIRE].

[55] D. Astefanesei, K. Goldstein and S. Mahapatra, Moduli and (un)attractor black hole thermodynamics, Gen. Rel. Grav. 40 (2008) 2069 [hep-th/0611140] [INSPIRE].

[56] M. Trigiante, Gauged supergravities, Phys. Rept. 680 (2017) 1 [arXiv:1609.09745] [INSPIRE].

[57] G. Dall'Agata, G. Inverso and M. Trigiante, Evidence for a family of $\mathrm{SO}(8)$ gauged supergravity theories, Phys. Rev. Lett. 109 (2012) 201301 [arXiv:1209.0760] [INSPIRE].

[58] A. Anabalón, D. Astefanesei, A. Gallerati and M. Trigiante, Hairy black holes and duality in an extended supergravity model, JHEP 04 (2018) 058 [arXiv:1712.06971] [INSPIRE].

[59] A. Anabalon and D. Astefanesei, Black holes in $\omega$-defomed gauged $N=8$ supergravity, Phys. Lett. B 732 (2014) 137 [arXiv:1311.7459] [INSPIRE].

[60] H. Lü, Y. Pang and C.N. Pope, An $\omega$ deformation of gauged STU supergravity, JHEP 04 (2014) 175 [arXiv:1402.1994] [INSPIRE].

[61] F. Faedo, D. Klemm and M. Nozawa, Hairy black holes in $N=2$ gauged supergravity, JHEP 11 (2015) 045 [arXiv: 1505.02986] [INSPIRE].

[62] U. Nucamendi and M. Salgado, Scalar hairy black holes and solitons in asymptotically flat space-times, Phys. Rev. D 68 (2003) 044026 [gr-qc/0301062] [InSPIRE].

[63] G.T. Horowitz, Introduction to holographic superconductors, Lect. Notes Phys. 828 (2011) 313 [arXiv: 1002.1722] [INSPIRE]. 